\title{
sciforum
}

\section{Effects of Serum 25-Hydroxyvitamin D concentration on Insulin Resistance and IVF-ET outcomes in PCOS}

\author{
Kun Liu ${ }^{a}$, César Martin Plagaro ${ }^{b}$, Bairong Shen ${ }^{c}$ \\ ${ }^{a}$ Reproductive Medicine Center of Lanzhou University First Hospital, Lanzhou, 730030, China \\ ${ }^{b}$ Biochemistry and Molecular Biology Department, University of the Basque Country UPV/EHU, \\ 48940, Leioa, Biscay, Spain. \\ ${ }^{C}$ Institutes for Systems Genetics, West China Hospital, Sichuan University, Chengdu, 610000, China
}

\section{Graphical Abstract}

None

Abstract.
Objective To explore the potential effects of
serum 25-hydroxyvitamin D on insulin
resistance and IVF pregnancy outcomes in the
patients with polycystic ovarian syndrome
(PCOS). Methods The patients with PCOS were
divided into vitamin D deficiency (VDD) group
and sufficient group according to the
concentration of serum vitamin D on GnRHa
administered day. To compare and analyze the
relationship between $25(\mathrm{OH}) \mathrm{D}$ and indicators of
insulin resistance and secretion function in
patients, and effects on pregnancy outcomes.
Results VDD group was more prone to have
high blood glucose $1 \mathrm{~h}$ post-meal,
hyperinsulinemia and insulin resistance, but with
significantly lower ISIcomp (P $<0.05$ ). G60 and
time-glycemic AUC has predictive value on
VDD in PCOS. Vitamin D sufficient is in favor
of improving the quality of embryos (r=0.3,
P=0.014), but does not affect IVF pregnancy
outcomes. Conclusion Vitamin D deficiency
was prevalent in PCOS and they were more
likely to have insulin regulatory dysfunction, but
it had no effect on IVF pregnancy rate.
[Key words] 25-hydroxyvitamin D; insulin
resistance; embryo; pregnancy

\section{Abstract.} serum 25-hydroxyvitamin D on insulin resistance and IVF pregnancy outcomes in the (PCOS). Methods The patients with PCOS were divided into vitamin D deficiency (VDD) group and sufficient group according to the concentration of serum vitamin D on GnRHa relationship between $25(\mathrm{OH}) \mathrm{D}$ and indicators of insulin resistance and secretion function in patients, and effects on pregnancy outcomes. Results VDD group was more prone to have high blood glucose $1 \mathrm{~h}$ post-meal, hyperinsulinemia and insulin resistance, but with significantly lower ISIcomp $(\mathrm{P}<0.05)$. G60 and time-glycemic AUC has predictive value on VDD in PCOS. Vitamin D sufficient is in favor of improving the quality of embryos $(r=0.3$, $\mathrm{P}=0.014)$, but does not affect IVF pregnancy outcomes. Conclusion Vitamin D deficiency was prevalen it had no effect on IVF pregnancy rate.

resistance; embryo; pregnancy 


\section{References}

[1] Abbasi F, Okeke Q, Reaven GM. Evaluation of fasting plasma insulin concentration as an estimate of insulin action in nondiabetic individuals: comparison with the homeostasis model assessment of insulin resistance (HOMAIR) [J]. Acta diabetologica. 2014,51(2):193-197.

[2] Bornstedt ME, Gjerlaugsen N, Olstad OK, Berg JP, Bredahl MK, Thorsby PM. Vitamin D metabolites influence expression of genes concerning cellular viability and function in insulin producing beta-cells (INS1E). Gene. 2020,746:144649.

[3] Visser JA. The Importance of Metabolic Dysfunction in Polycystic Ovary Syndrome.[J]. Nat Rev Endocrinol. 2021,17(2):77-78.

[4] Ozegowska K, Korman M, Szmyt A, Pawelczyk L. Heterogeneity of Endocrinologic and Metabolic Parameters in Reproductive Age Polycystic Ovary Syndrome (PCOS) Women Concerning the Severity of Hyperandrogenemia-A New Insight on Syndrome Pathogenesis [J]. Int J Environ Res Public Health. 2020,17(24):9291.

[5] Mishra AK, Pandey A, Gautam DK. A study of Serum Vitamin D and Insulin Resistance in Subjects with Prediabetes [J]. J Assoc Physicians India. 2020,68(1):47.

[6] Vulcan T, Filip GA, Lenghel LM, Suciu T, Ilut P, Procopciuc LM. Polymorphisms of Vitamin D Receptor and the Effect on Metabolic and Endocrine Abnormalities in Polycystic Ovary Syndrome: A Review. [J]. Horm Metab Res. 2021,53(10):645-653.

[7] Gupta T, Rawat M, Gupta N, Arora S. Study of Effect of Vitamin D Supplementation on the Clinical, Hormonal and Metabolic Profile of the PCOS Women [J]. J Obstet Gynaecol India. 2017,67(5):349-355.

[8] Bornstedt ME, Gjerlaugsen N, Pepaj M, Bredahl MKL, Thorsby PM. Vitamin D Increases Glucose Stimulated Insulin Secretion from Insulin Producing Beta Cells (INS1E) [J]. Int J Endocrinol Metab. 2019,17(1): e74255.

[9] Rojas J, Bermudez V, Palmar J, et al. Pancreatic Beta Cell Death: Novel Potential Mechanisms in Diabetes Therapy [J]. J Diabetes Res. 2018:9601801.

[10] Guo J, Liu S, Wang P, Ren H, Li Y. Characterization of VDR and CYP27B1 expression in the endometrium during the menstrual cycle before embryo transfer: implications for endometrial receptivity [J]. Reprod Biol Endocrinol. 2020,18(1):24.

[11] Bøllehuus Hansen L, Rehfeld A, de Neergaard R, et al. Selection of High-Quality Spermatozoa May Be Promoted by Activated Vitamin D in the Woman [J]. J Clin Endocrinol Metab. 2017,102(3):950-961.

[12] A Arnanz, ND Munck, IE Khatib et al. Vitamin D in Follicular Fluid Correlates With the Euploid Status of Blastocysts in a Vitamin D Deficient Population [J]. Front Endocrinol (Lausanne). 2021,11:609524.

[13] Antunes RA, Mancebo ACA, Reginatto MW, et al. Lower follicular fluid vitamin D concentration is related to a higher number of large ovarian follicles [J]. Reprod Biomed Online. 2018,36(3):277-284.

[14] Ciepiela, Przemysaw , Dulba, et al. Vitamin D as a follicular marker of human oocyte quality and a serum marker of in vitro fertilization outcome [J]. J Assist Reprod Genet. 2018,35(7):1265-1276.

[15] YF Ruelas, MDT Equihua, NAJ Solis, et al. Vitamin D status and its relation to insulin resistance in a Mexican pediatric population [J]. J Pediatr Endocrinol Metab. 2020,33(4):481-486. 